Japanese Journal of Applied Physics, 26 (4) (1987) 578-581

\title{
Fluorescence from Pearls to Distinguish M other Oysters Used in Pearl Culture
}

Tadaki Miyoshi, Yasunori Matsuda ${ }^{1}$ and Hiroshi Komatsu ${ }^{1}$

Technical College, Yamaguchi U niversity, Tokiwadai, U be, Yamaguchi 755

${ }^{1}$ Pearl Research laboratory, K. MIKIMOTO \& Co., Ltd., Aobadai, Meguro-ku, Tokyo 153

(Received October 13, 1986; accepted January 24, 1987)

The fluorescence spectra of pearls and shells of Pteria penguin (mabe), Pinctada fucata (Japan's Akoya oyster) and Pinctada maxima (yellow lip oyster) have been measured in order to distinguish species of the mother oyster which produce that pearl. A distinction is possible for these pearls using differences in the fluorescence spectra. The spectral difference has been attributable to colouring matter or screloprotain in pearls and shells. 


\section{$\S 1$. Introduction}

Several mother oysters are used in pearl culturing: seawater bivalves (Pinctada fucata (Japan's Akoya oyster), Pinctada maxima (yellow lip oyster), Pinctada margaritifera (black lip oyster) and Pteria penguin (mabe)), seawater gastropod (genus Haliotis (abalone)) and freshwater bivalves (Hyriopsis shlegeli et al.). Nondestructive methods for distinguishing mother oysters have been important from practical points of view since the commercial value of pearls depends on the mother oysters. In summary, freshwater pearls can be distinguished from seawater pearls by using X-ray fluorescence analysis, since freshwater pearls contain a large amount of manganese. ${ }^{1)}$ Pearls of P. margaritifera show a peculiar absorption at $700 \mathrm{~nm}^{2}{ }^{2}$ Pearls of genus Haliotis can be distinguished from pearls produced from any other mother oysters under a microscope since the surface of gastropod nacre shows a pyramid stack formation. ${ }^{3)}$ Here, nacre is the surface layer part of a pearl and a shell.

In a previous paper, we reported that a distinction is possible for pearls of $\mathrm{P}$. fucata and P. maxima, using differences in the fluorescence spectra under $\mathrm{N}_{2}$ laser excitation. ${ }^{4}$ On the other hand, as far as we know, there has been no report on the distinction of mother oysters for P. penguin. In this work, we measured the fluorescence spectra of pearls and shells of $P$. penguin, P. fucata and P. maxima under $\mathrm{N}_{2}$ laser and ordinary lamp excitation in order to apply the fluorescence method to the distinction of these pearls.

\section{§2. Experimental Procedure}

Laser-induced fluorescence has been measured at room temperature using the following apparatus. The excitation source was a pulsed $\mathrm{N}_{2}$ laser (NDC JS-1000L: $\lambda=$ $337.1 \mathrm{~nm}$, pulse duration $=5 \mathrm{~ns}$, repetition rate $=4 \mathrm{~Hz}$ ). The laser beam was set at an angle of about $50^{\circ}$ off the normal incidence to the plane of the sample and was focused on a spot about $1 \mathrm{~mm}^{2}$ in area by a quartz lens (focal length $\mathrm{f}=150 \mathrm{~mm}$, diameter $\mathrm{d}=$ $40 \mathrm{~mm}$ ). The peak power density of the laser light on a sample was regulated to be about $50 \mathrm{~kW} / \mathrm{cm}^{2}$ using neutral density filters. The sample was held between $\mathrm{Cu}$ and an Al plates with a hole $6 \mathrm{~mm}$ in diameter. The laser beam and fluorescence passed through the hole. Fluorescence was observed at $90^{\circ}$ to the laser beam and was focused on the 
entrance slit of a $50 \mathrm{~cm}$ monochromator (Oyo Bunko ASI-50S) by a glass lens ( $\mathrm{f}=70$ $\mathrm{mm}, \mathrm{d}=40 \mathrm{~mm}$ ). A glass filter (Toshiba L-39) was used in order to cut off any scattered light of the laser beam. Time-integrated and time-resolved fluorescence spectra were measured with the monochromator, a photomultiplier (Hamamatsu R955), a boxcar integrator (NF Circuit Design Block BX-531) and a recorder. The transient characteristics of fluorescence were measured using a storage oscilloscope (Iwatsu Electric TS-8123). Ordinary fluorescence has also been measured using a spectrofluorophotometer (Japan Spectroscopic FP-770) to examine the effect of the excitation source on the fluorescence spectra. The apparatus constant is not corrected for the ordinary fluorescence spectra.

The materials in this study were the shells of P. penguin as well as the shells and pearls of P. fucata and P. maxima. Mother oyster of P. penguin produces various pearl colours: reddish brown, pink and white. Reddish brown is caused by colouring matter in nacre and pink is caused by an interference effect in nacre. In this experiment, we adopt the nacre of shell instead of pearl for the following reasons: nacre of shell has the same chemical composition as a pearl itself and the pearl of P. penguin is hard to obtain commercially since the amount produced is very small. Although we could not obtain the pearl of $P$. penguin, nacre of shell would show a similar fluorescence spectrum to that of the pearl. The spectrum similarity was confirmed for P. fucata and P. maxima (Figs. 1 and 5).

Mother oysters of P. fucata produce various pearl colours: white, green, pink, yellow and blue. Green and pink are caused by an interference effect in nacre; while yellow and blue are considered to be due to organic substances in the pearls. Mother oyster of $P$. maxima produces white and yellow pearls. The yellow appearance is also considered to be due to organic substances. In this experiment, white and yellow pearls and shells of these two species have been investigated.

Conchiolin, which is a kind of scleroprotain in nacre for P. fucata and P. maxima, was investigated. Nacre was completely decalcified with an EDTA (ethylendiamine tetraacetic acid) solution; then, nacreous conchiolin was obtained as an insoluble residue. We adopt nacre of shell instead of pearl since it has the same chemical composition as a pearl itself (as described above). 


\section{§3. Results and Discussion}

\subsection{P. penguin}

Figure 1 shows the time-integrated fluorescence spectra of nacre of shells of pearl oysters: reddish brown and white shells of P. penguin, a white shell of P. maxima and a yellow shell of P. fucata. Three peaks were observed at about $440 \mathrm{~nm}, 540 \mathrm{~nm}$ and 620 $\mathrm{nm}$ in the reddish-brown shell of P. penguin. Peaks at 540 and $620 \mathrm{~nm}$ were not observed in the shells of P. maxima and P. fucata. Thus, a distinction is possible for reddish-brown pearls of $P$. penguin from pearls of $P$. fucata and P. maxma using this difference in the fluorescence spectra. On the other hand, the 540 and $620 \mathrm{~nm}$ peaks were not observed in the white shell of P. penguin. The fluorescene spectrum of the white shell is similar to that of P. maxima.

Since the fluorescence intensity of the $620 \mathrm{~nm}$ peak is considered to depend on the wavelength of the excitation light, the excitation spectrum is measured by using a spectrofluorophotometer. Figure 2 shows the excitation spectra of the 620 and $540 \mathrm{~nm}$ peaks of the reddish-brown shell. In the excitation spectrum, the observed wavelength was fixed at $620 \mathrm{~nm}$ (upper curve) or $540 \mathrm{~nm}$ (lower curve) and the excitation wavelength was scanned. The abscissa represents the excitation wavelength. A peak was observed at about $400 \mathrm{~nm}$. Thus, $400 \mathrm{~nm}$ light excitation may be most favourable for an observation of the $620 \mathrm{~nm}$ peak. Figure 3 shows ordinary fluorescence spectra of reddish-brown and white shells under 400 and $350 \mathrm{~nm}$ light excitation using the spectrofluorophotometer. The $620 \mathrm{~nm}$ peak was observed even in the white shell under $400 \mathrm{~nm}$ excitation. The pink shell of $\mathrm{P}$. penguin shows a similar fluorescence spectrum to that of the white shell. On the other hand, the $620 \mathrm{~nm}$ peak was not observed in the nacre of shells and pearls of P. fucata and P. maxima under $400 \mathrm{~nm}$ excitation. Thus, a distinction is possible for pearls of $P$. penguin with any colours using the fluorescence spectra under $400 \mathrm{~nm}$ excitation.

The fluorescence intensity of the $620 \mathrm{~nm}$ peak depends on the colour of shells. This suggests that the $620 \mathrm{~nm}$ peak can be attributed to colouring matter, which causes a reddish-brown appearance of the shell. Time-resolved fluorescence spectra were measured in order to examine the properties of the colouring matter. Figure 4 shows the 
time-resolved fluorescence spectra of the reddish-brown shell of $P$. penguin under $\mathrm{N}_{2}$ laser excitation. While the relative intensity of the $620 \mathrm{~nm}$ peak is weak at short delay time, it increases with an increasing delay time. This result suggests that the $620 \mathrm{~nm}$ peak has a long decay time. The decay time was determined from the transient characteristics of the fluorescence measured with a storage oscilloscope. The decay time is about $4 \mathrm{~ns}$ for both the $440 \mathrm{~nm}$ peak and the $540 \mathrm{~nm}$ peak and about $12 \mathrm{~ns}$ for the 620 $\mathrm{nm}$ peak. This result is consistent with that of the time-resolved fluorescence spectra. It is reported that porphyrin is contained in shells of $P$. penguin $;^{5)}$ it shows a fluorescence peak at about $620 \mathrm{~nm}$ and an absorption peak at about $400 \mathrm{~nm}^{6}{ }^{6}$ The decay time of fluorescence is $11 \mathrm{~ns}{ }^{7)}$ These results indicate that the $620 \mathrm{~nm}$ peak is caused by porphyrin.

The fluorescence intensity of the $540 \mathrm{~nm}$ peak depends on both the colour of shells and on the wavelength of excitation light (Figs. 2 and 3). This behaviour is similar to that of the $620 \mathrm{~nm}$ peak. However, the $540 \mathrm{~nm}$ peak is not so intense as the $620 \mathrm{~nm}$ peak under the $400 \mathrm{~nm}$ excitation. Moreover, the decay time of the $540 \mathrm{~nm}$ peak is different from that of the $620 \mathrm{~nm}$ peak. Thus, the $540 \mathrm{~nm}$ peak is considered to be due to other colouring matter. It is reported that carotenoids show a fluorescence peak at about $\mathbf{5 5 0}$ $\mathrm{nm}$ and that they are in marine shells such as shell-cameos, Chlamys nobilis, Pecten varius et al. ${ }^{8)}$ On the basis of this result, we consider that the $540 \mathrm{~nm}$ peak is caused by carotenoids.

\subsection{P. fucata and P. maxima}

Time-integrated fluorescence spectra of pearls of P. fucata and P. maxima were reported in a previous paper. ${ }^{4}$ Since pearls of $P$. fucata show a peak at a longer wavelength than that of P. maxima (Fig. 5), a distinction is possible by using this difference. It has been interpreted that the spectral difference observed in pearls of $P$. fucata and P. maxima is caused by the difference in conchiolin, which is a kind of scleroprotain in pearls and shells.

In this paper, fluorescence from conchiolin extracted from nacre was measured for $P$. fucata and P. maxima in order to examine the validity of the interpretation above. Nacre consists of aragonite $\left(\mathrm{CaCO}_{3}\right)$, conchiolin and other trace components. The ratio of 
aragonite and conchiolin is about 95:5 (w/w). A single crystal of aragonite is enclosed with conchiolin sheets and thousands of these piles build the nacreous structure like a brick wall. Conchiolin is a binding material between aragonite crystals. Figure 6 shows the fluorescence spectra of conchiolin, aragonite and mixture of these materials for $P$. fucata (solid curves) and P. maxima (dashed curves). Aragonite is powdered ore, which is collected in the Aragon province of Spain. The peak wavelength of fluorescence from conchiolin for P. fucata is longer than that for P. maxima and the fluorescence spectra of a mixture of aragonite and conchiolin in the ratio of 93:7 are in qualitative agreement with that in Fig. 5. Thus, the result in Fig. 6 supports the interpretation that the spectral difference in Fig. 5 is due to a difference in conchiolin.

Time-resolved fluorescence spectra were measured in order to examine the properties of conchiolin. Figure 7 shows the time-resolved fluorescence spectra of the white pearls of P. fucata (solid curves) and P. maxima (dashed curves) under $\mathrm{N}_{2}$ laser excitation. Fluorescence peaks for both pearls shift to longer wavelengths with an increasing delay time. The time-resolved fluorescence spectra of conchiolin also show similar results. These results suggest that there are two groups of components in pearls. Group A shows fluorescence with a shorter decay time and a shorter wavelength peak, while group B shows that with a longer decay time and a longer wavelength peak. The ratio A/B may be large in pearls of P. maxima, so that they show fluorescence with a shorter wavelength peak. On the other hand, the ratio A/B may be small in pearls of P. fucata, so that they show fluorescence with a longer wavelength peak. The decay time was determined from the transient characteristics of fluorescene measured with the storage oscilloscope. The decay time is about $4 \mathrm{~ns}$ for $\lambda \leq 470 \mathrm{~nm}$ and about $5 \mathrm{~ns}$ for $\lambda \geq 500$ $\mathrm{nm}$ for both pearls. This result is consistent with the two group model above.

The fluorecence spectra of pearls of P. fucata have been measured using an $\mathrm{Hg}$ lamp as the excitation source. ${ }^{9)}$ The peak wavelength of the fluorescence depends on the colour appearances; it appears at $470 \mathrm{~nm} \leq \lambda \leq 530 \mathrm{~nm}$. This result is in qualitative agreement with the present result shown in Fig. 5, while the peak wavelength of the former is longer than that of the latter. This difference is considered to be due to the difference in the excitation wavelength.

In summary, the fluorescence spectra of pearls and shells of mother oysters have been 
measured under $\mathrm{N}_{2}$ laser and ordinary lamp excitation. A distinction is possible for pearls of $P$. penguin using the peculiar peak at $620 \mathrm{~nm}$, which is attributable to porphyrin in nacre. A distinction is also possible for pearls of P. fucata and P. maxima using the difference in the fluorescence spectra. The spectral difference is considered to be attributable to the difference in conchiolin. 


\section{References}

1) Y. Horiguchi: Bull. Jpn. Soc. Sci. Fish. 25 (1959) 392 [in Japanese].

2) H. Komatsu and S. Akamatsu: J. Gemmological Soc. Jpn. 5 (1979) No. 4, 3 [in Japanese].

3) S. W. Wise: Eclogae. Geol. Helv. 63 (1970) 775.

4) T. Miyoshi, Y. Matsuda and H. Komatsu: Jpn. J. Appl. Phys. 25 (1986) 1606.

5) R. Tixier: Ann. L'Inst. Oceanographique 22 (1945) 343.

6) J. E. Falk: Porphyrins and Metalloporphyrins (Elsevier, Amsterdam, 1964) p. 87.

7) Y. Onoue, K. Hiraki and Y. Nishikawa: Bunseki Kagaku 31 (1982) 169 [in Japanese].

8) M. L. Dele-Dubois, J. C. Merlin and J. P. Poirot: J. Gemmological Soc. Jpn. 8 (1981) 161.

9) Y. Sawada: Bull. Natl. Pearl Res. Lab. 4 (1958) 340 [in Japanese]. 


\section{Figure captions}

Fig. 1. Time-integrated fluorescence spectra of nacre of shells of several mother oysters at $300 \mathrm{~K}$ under $\mathrm{N}_{2}$ laser excitation. Peak intensities are normalized.

Fig. 2. Excitation spectra of the $620 \mathrm{~nm}$ peak and the $540 \mathrm{~nm}$ peak of the reddish-brown shell of $P$. penguin.

Fig. 3. Ordinary fluorescence spectra of reddish-brown and white shells of $P$. penguin under 400 and $350 \mathrm{~nm}$ light excitation. Peak intensities are normalized.

Fig. 4. Time-resolved fluorescence spectra of the reddish-brown shell of $P$. penguin. Intensity ratios of the $620 \mathrm{~nm}$ peak are as follows: 0.6 (delay time $\mathrm{t}=-2 \mathrm{~ns}), 1(8 \mathrm{~ns})$, 0.3 (18 ns). The inset shows transient characteristics of the laser pulse.

Fig. 5. Time-integrated fluorescence spectra of yellow and white pearls of P. fucata (solid curve) and P. maxima (dashed curve). Peak intensities are normalized.

Fig. 6. Time-integrated fluorescence spectra of conchiolin, aragonite and mixtures of these materials. The percentage of conchiolin is represented by weight percent. Conchiolin is extracted from nacre of P. fucata (solid curves) and P. maxima (dashed curves). Peak intensities are normalized.

Fig. 7. Time-resolved fluorescence spectra of the white pearls of P. fucata (solid curves) and P. maxima (dashed curves). Peak intensity ratios are as follows: 1 (delay time $t=$ $-2 \mathrm{~ns}), 1$ (8 ns), 0.3 (18 ns). 
Fig. 1

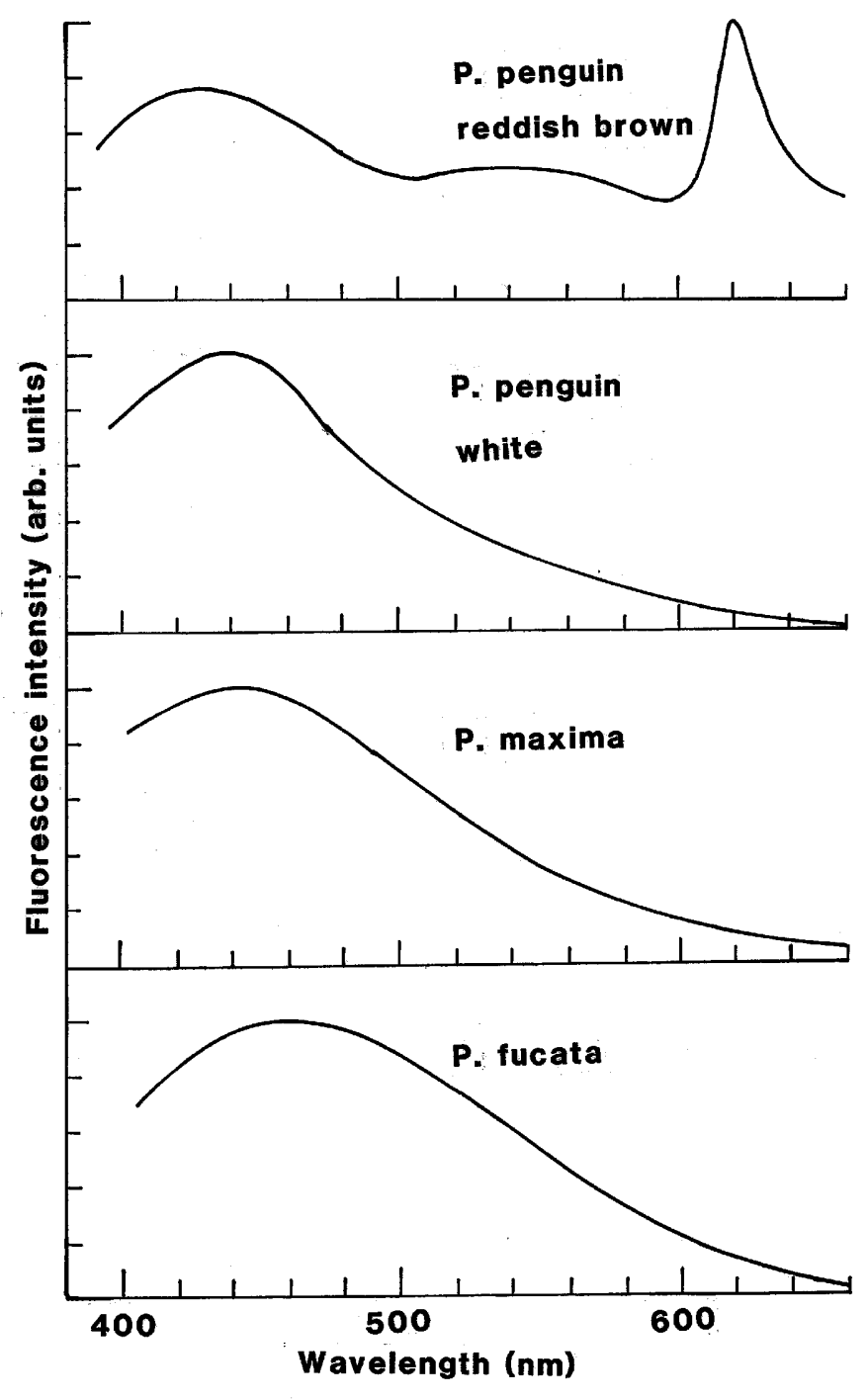

Fig. 2

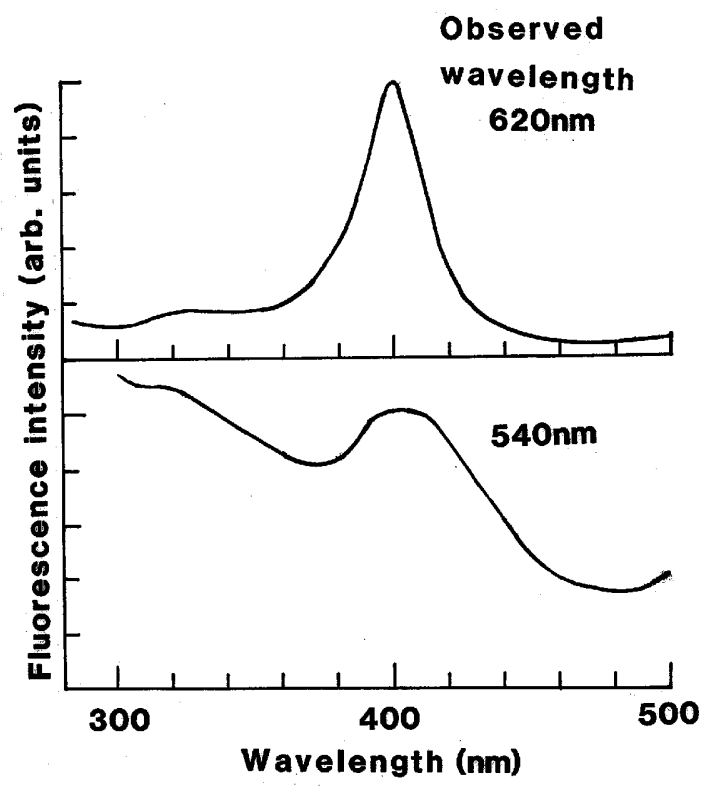


Fig. 3

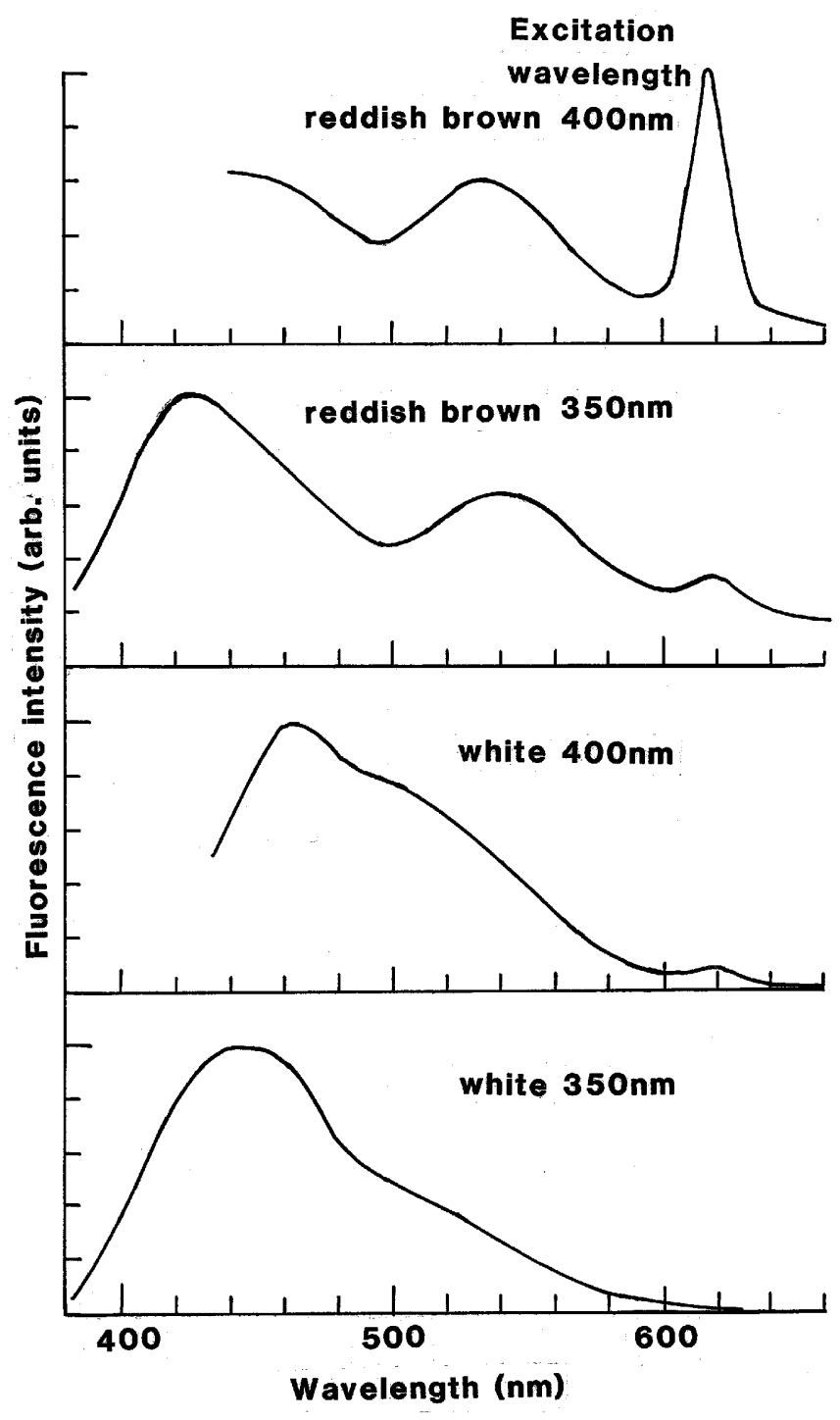


Fig. 4

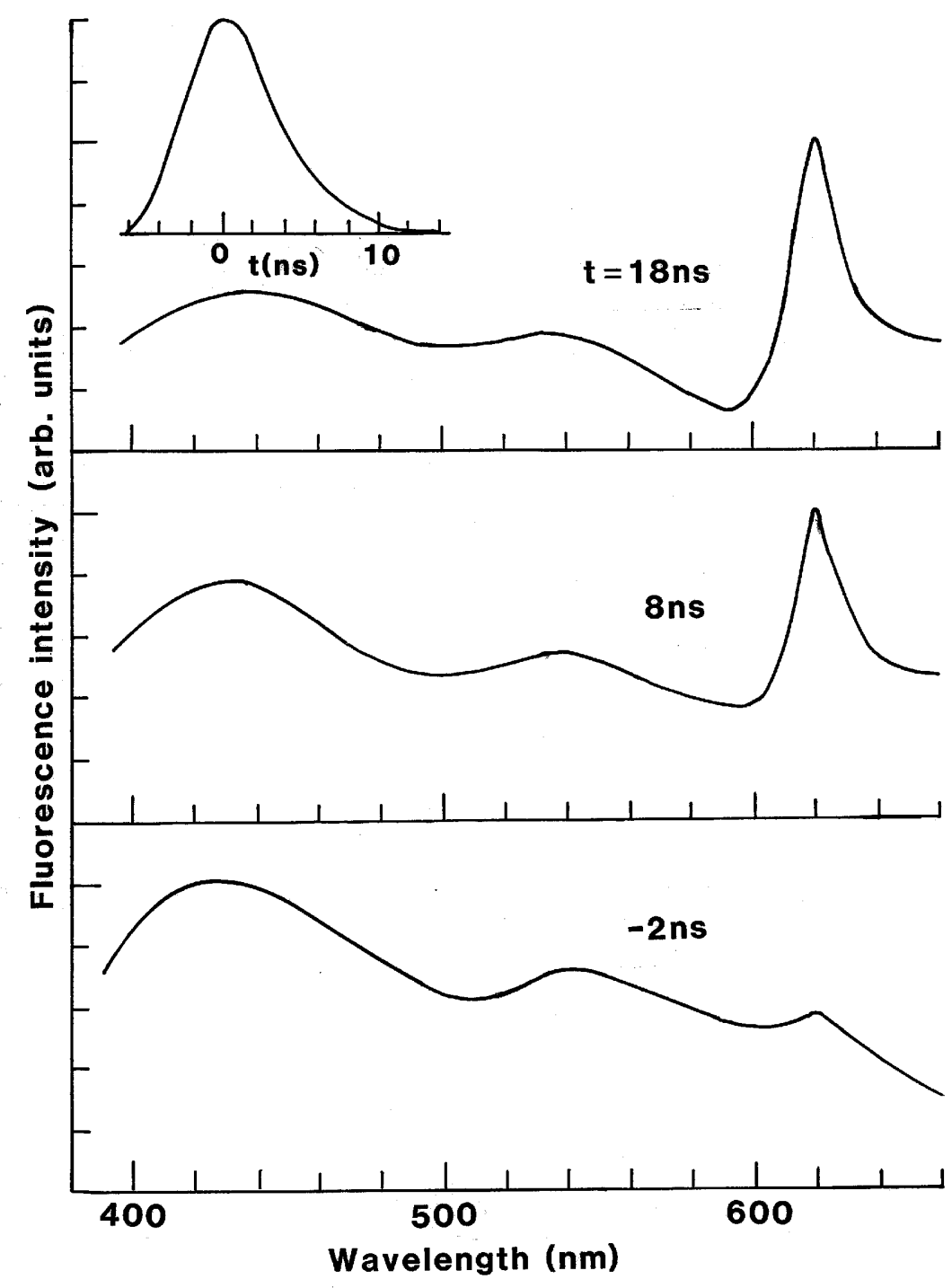

Fig. 5

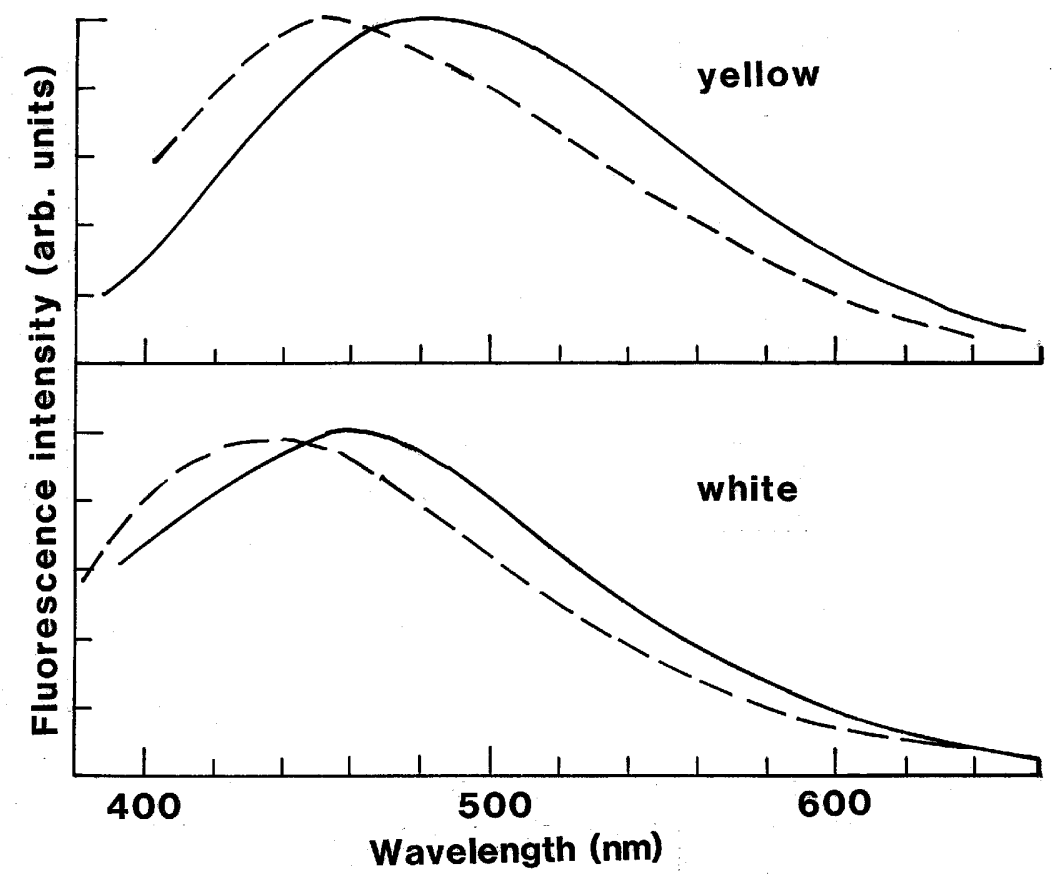


Fig. 6

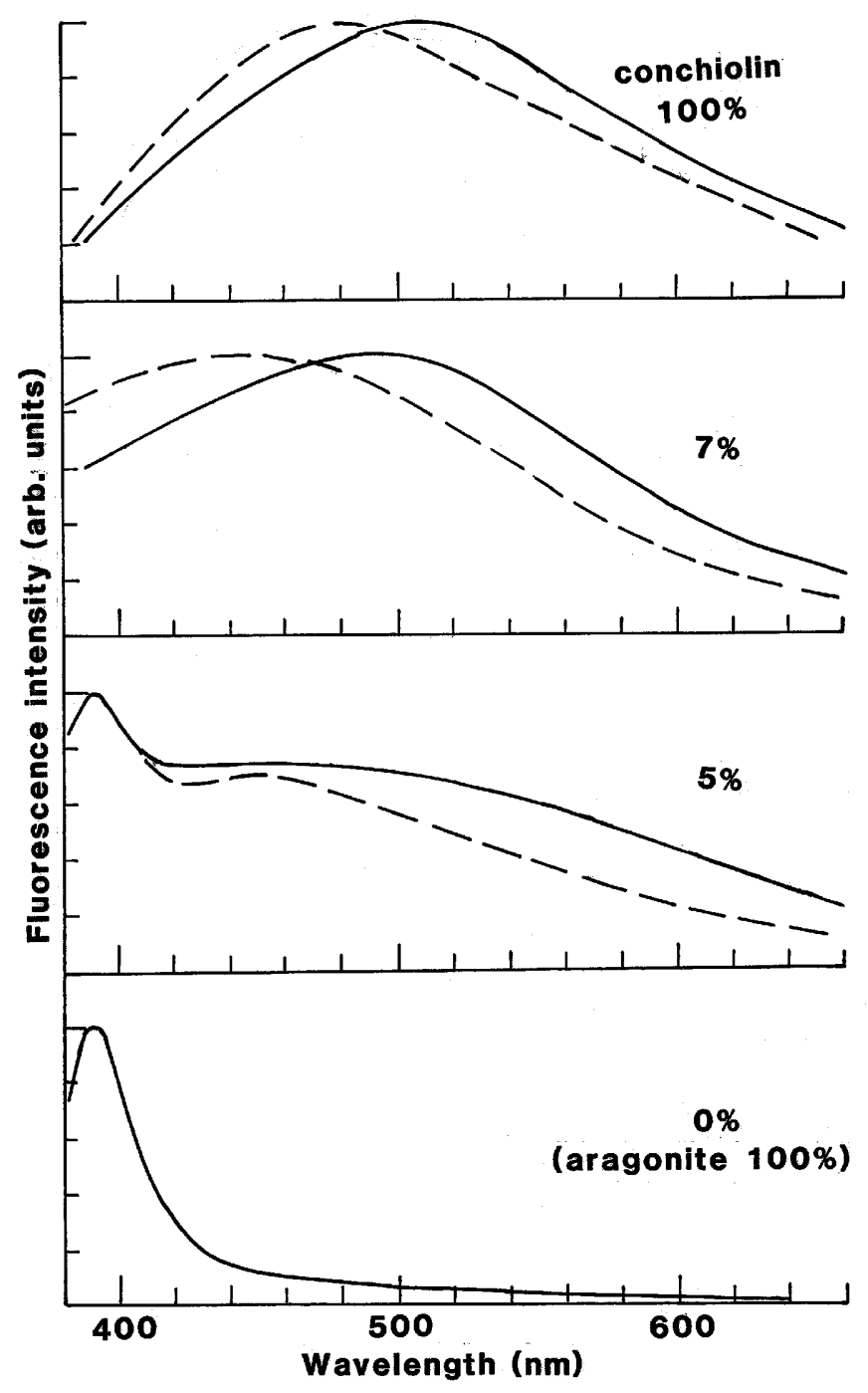

Fig. 7

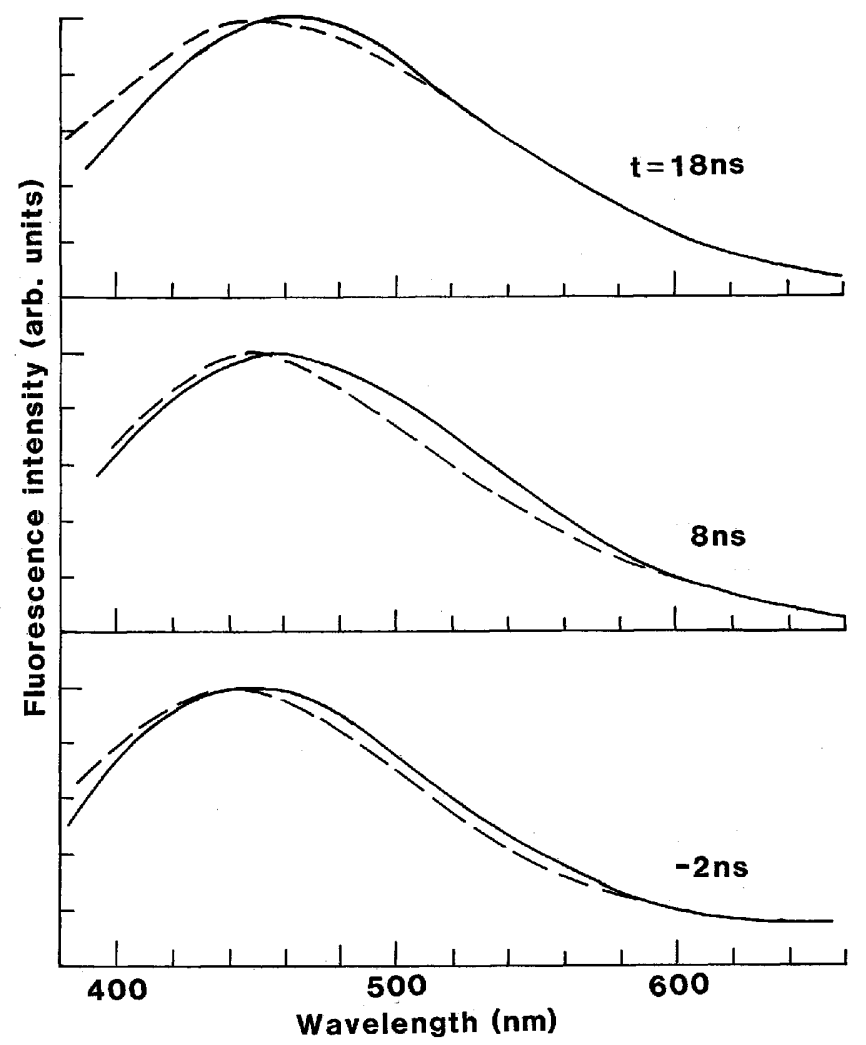

